\title{
Olanzapine (5 mg) Plus Standard Triple Antiemetic Therapy for the Prevention of Multiple-Day Cisplatin Chemotherapy-Induced Nausea and Vomiting: A Prospective Randomized Controlled Study
}

Jiali Gao

Ordos Central Hospital

Jun Zhao

Ordos Central Hospital

Caihong Jiang

Ordos Central Hospital

Feng Chen

Ordos Central Hospital

Lanzhen Zhao

Ordos Central Hospital

Ying Jiang

Ordos Central Hospital

Hui Li

Ordos Central Hospital

Wenjuan Wang

Ordos Central Hospital

Yungaowa Wu

Ordos Central Hospital

Yilan Jin

Ordos Central Hospital

Lenggaowa Da

Ordos Central Hospital

Guang Liu

Ordos Central Hospital

Yajuan Zhang

Ordos Central Hospital

Hongxia Li

Ordos Central Hospital

Zewei Zhang

Sun Yat-sen University Cancer Center 


\section{Gaowa Jin}

Ordos Central Hospital

\section{Quanfu Li ( $\sim 1729259137 @ q q . c o m$ )}

Ordos Central Hospital

\section{Research Article}

Keywords: Olanzapine, Aprepitant, CINV, Multiple-day cisplatin chemotherapy

Posted Date: August 23rd, 2021

DOl: https://doi.org/10.21203/rs.3.rs-557438/v1

License: (c) (1) This work is licensed under a Creative Commons Attribution 4.0 International License. Read Full License 


\section{Abstract}

Objective A prospective randomized controlled trial was conducted to compare $5 \mathrm{mg}$ olanzapine plus standard triple antiemetic therapy for the prevention of nausea and vomiting induced by multiple-day cisplatin chemotherapy.

Methods Patients received 3-day cisplatin-based chemotherapy $(25 \mathrm{mg} / \mathrm{m} 2 / \mathrm{d})$ were given either $5 \mathrm{mg}$ olanzapine quadruple therapy (aprepitant, tropisetron, dexamethasone) or $5 \mathrm{mg}$ olanzapine-based triplet therapy. The primary end-point was the complete response(CR) in the overall phase $(O P)(0-120 \mathrm{~h})$ between quadruple regimen group and triplet regimen group. The secondary end-points were the $\mathrm{CR}$ in the acute phase(AP)(0-24h), delayed phase(DP)(25-120h) between two groups. The first time of vomiting was also compared by Kaplan-Meier curves. The impact of chemotherapy induced nausea and vomiting(CINV) on the quality of life was assessed by the Functional Living Index-Emesis(FLIE). Aprepitant-related adverse effects (AEs) was also recorded.

Results (1) The primary end-point CR during overall phase was $76.0 \%$ (45/59) vs $67.0 \%(41 / 61)$ between the quadruple regimen group and triplet regimen group $(P=0.271)$. The secondary end-point $\mathrm{CR}$ during the AP was significantly higher in the quadruple group than in the triple group, which was $100.0 \%(59 / 59)$ vs $93.0 \%(57 / 61)(P=0.045)$. The difference between the groups was especially greater in the delayed phase(quadruple group $76.0 \%(45 / 59)$ vs triple group $67.0 \%(41 / 61)(P=0.271))$. The rate of patients who achieved total protection in the overall phase was also larger in the quadruple group than in the triple group(28.8\% (17/59) vs $23.0 \%(14 / 61)(P=0.463))$. During the OP, the incidence of no vomiting in quadruple group and triple group was $93.2 \%(55 / 59)$ vs $80.3 \%(49 / 61)(P=0.038)$ respectively.(2) KaplanMeier curves of time to first emesis were obviously longer in the quadruple group than in the triple group $(P=0.031)$. According to FLIE, no impact of CINV on daily life was defined as total score of questionnaire $>108$, this study exhibited identical life quality in quadruple group and the triplet group.(3) The most common aprepitant- and olanzapine-related AEs included sedation, fatigue and constipation. The occurrences between two groups were identical.

Conclusion It may been recommended that combined $5 \mathrm{mg}$ olanzapine with aprepitant, tropisetron, dexamethasone quadruple therapy for the prevention of multiple-day cisplatin induced nausea and vomiting due to the better CINV control rate and safety.

\section{Introduction}

Patients receiving multiple-day cisplatin chemotherapy are at risk of both acute and delayed nausea and vomiting for each day, as acute and delayed emesis may overlap after the initial day chemotherapy until the last day of chemotherapy ${ }^{[1,2]}$. Although the combination of aprepitant, 5-HT3 receptor antagonist(5HT3RA) and dexamethasone(DXM) had showed higher complete response than the combination of 5HT3RA plus dexamethasone in cisplatin multiple-day chemotherapy clinical studies, nausea remains a major problem for many patients ${ }^{[3,4,5]}$. The activity of olanzapine on multiple receptors, particularly the 
D2, 5-HT2C and 5-HT3 receptors, may be involved in nausea and vomiting. A single-institution phase 3 trial showed that olanzapine was comparable to aprepitant in the control of CINV, and nausea was better controlled with olanzapine in delayed period and overall period ${ }^{[6]}$.Somnolence is a major side effect when olanzapine was administered at a dose of $10 \mathrm{mg}$. In a phase 2 study, $5 \mathrm{mg}$ olanzapine has shown equivalent activity to $10 \mathrm{mg}$ olanzapine and a favourable safety in relation to somnolence ${ }^{[7]}$. Guidelines suggest that a dose reduction to $5 \mathrm{mg}$ should be considered to prevent sedation, and a randomised, double-blind, placebo-controlled phase 3 study showed that $5 \mathrm{mg}$ olanzapine combined with aprepitant, palonosetron and dexamethasone could be a new standard antiemetic therapy for patients undergoing cisplatin single-day chemotherapy ${ }^{[8]}$. Aprepitant is an oral neurokinin-1 receptor antagonist ${ }^{[9]}$. A randomized controlled trial was conducted to compare the efficacy of $5 \mathrm{mg}$ olanzapine plus triplet therapy (aprepitant, tropisetron, dexamethasone) versus $5 \mathrm{mg}$ olanzapine-based triplet therapy in preventing CINV in patients receiving multiple-day cisplatin chemotherapy(chiCTR1800018424).

\section{Methods}

\section{Patients}

This study was approved by the local Institutional Review Board, and all patients provided written informed consent before the start of study procedures. A randomized, clinical trial (chiCTR1800018424) was conducted to compare the effectiveness of $5 \mathrm{mg}$ olanzapine plus triplet therapy (aprepitant, tropisetron, dexamethasone) (Quadruple group ) versus $5 \mathrm{mg}$ olanzapine-based triplet therapy(Triplet group) in preventing CINV in patients receiving multiple-day cisplatin chemotherapy.

\section{End points}

We chose the $\mathrm{CR}$ rate, defined as the absence of emetic episodes and no use of rescue medications during the overall phase after the initiation of cisplatin, as the primary end-point. Secondary end-points are the CR rate in the acute phase and the delayed phase. The total control(TC) rate is defined as the absence of nausea and emetic episodes and no use of rescue medications for the acute, delayed, and overall phase. We used a $100 \mathrm{~mm}$ categorical scale to stratify nausea and chose $\leq 5 \mathrm{~mm}$ to define the total control. The time of treatment failure is defined as the time of first emetic episode or the use of rescue medication. AEs were graded according to Common Terminology Criteria for Adverse Events (CTCAE) V.4.0.

\section{Randomization}

After confirming that the patients fulfills the eligibility criteria, the patients are randomized by random digits table.

\section{Eligibility criteria}

Inclusion criteria 
(1) Patients older than 18 years who will receive 3-day cisplatin-based chemotherapy $(25 \mathrm{mg} / \mathrm{m} 2 / \mathrm{d})$

enrolled in the study. All patients had histologically confirmed, (2) Karnofsky Performance Scale $\geq 70$, (3) There is no abnormality in liver and kidney function, blood routine and electrocardiogram before chemotherapy, including: White Blood Cell Couct $>3.5 \times 10^{\wedge} 9 / \mathrm{L}$, Absolute neutrophil count $>1.5 \times 10^{\wedge} 9 / \mathrm{L}$, Platelet count $>85 \times 10^{\wedge} 9 / \mathrm{L}$, Alkaline phosphatase $<2.5$ upper limit of normal (ULN), alanine transaminase $<2.5$ upper limit of normal (ULN), Bilirubin <1.5 ULN, Creatinine <1.5 ULN, (4) Patients without chemotherapy contraindications after CT and MRI examination, (5) No episodes of nausea and vomiting occurred during last 1 week before enrollment, and no aprepitant or olanzapine was used for pretreatment, (6) Patients are able to understand and describe patient-reported outcomes.

\section{Exclusion criteria}

Patients were excluded if they meet any of the following criterias: (1)Symptomatic brain metastases, (2) Requiring treatment for ascites or pleural effusion, (3) Requiring radiotherapy in the abdominal or pelvic field, (4) Requiring anticonvulsant medication, (5)History of hypersensitivity or allergy to the study drugs or similarcompounds, (6) Severe complications (pulmonary fibrosis, heart failure, myocardialinfarction, unstable angina, cerebral vascular disorder, psychiatricdisease, renal dysfunction, liver dysfunction, intestinalparesis, ileus, uncontrol lable diabetes mellitus, active peptic ulceretc), (7)History of using any of the following drugs within 48h, opioids, aprepitant, 5-HT3 receptor antagonists, dexamethasone, dopamine receptor antagonists, antihistamines, benzodiazepines and phenothiazine antipsychotics, (8)Pregnant or lactating women or women with child bearing potential, or men wishing to be the father of children, (9) Partial/complete bowel obstruction, (10) Malignant tumor of digestive tract,

\section{Treatments}

The study antiemetic administrations are shown in Table 1. Patients in quadruple antiemetic regimen group received: Aprepitant $125 \mathrm{mg}$ po day 1, 80mg po days 2-3(EMEND, MSD Sharp \& Dohme, Haar, Germany), Olanzapine 5mg po days 1-3, tropisetron 5mg iv days 1-3 (Beijing Shuanglu Pharmaceutical Co. Ltd., China), dexamethasone $5 \mathrm{mg}$ iv days 1-3. Patients in triple antiemetic regimen group received: Olanzapine $5 \mathrm{mg}$ po days 1-3, tropisetron $5 \mathrm{mg}$ iv days 1-3, dexamethasone $10 \mathrm{mg}$ iv days 1-3. The aprepitant group had a half dosage of dexamethasone besides tropisetron hydrochloride and aprepitant, since the function of CYP3A4 in DXM pharmacokinetics could be exhibited by aprepitant ${ }^{[10]}$.

Table 1 Antiemetic administrations 


\begin{tabular}{|c|c|c|c|}
\hline & Day1 & Day2 & Day3 \\
\hline \multirow{5}{*}{ Quadruple antiemetic regimen } & Aprepitant $125 \mathrm{mg}$ po & Aprepitant $80 \mathrm{mg}$ po & Aprepitant $80 \mathrm{mg}$ po \\
\hline & Olanzapine 5mg po & Olanzapine $5 \mathrm{mg}$ po & Olanzapine $5 \mathrm{mg}$ po \\
\hline & Tropisetron 5mg iv & Tropisetron $5 \mathrm{mg}$ iv & Tropisetron 5mg iv \\
\hline & Dexamethasone & Dexamethasone & Dexamethasone \\
\hline & $5 \mathrm{mg}$ po & $5 \mathrm{mg}$ po & $5 \mathrm{mg}$ po \\
\hline \multirow[t]{4}{*}{ Triple antiemetic regimen } & Olanzapine 5mg po & Olanzapine $5 \mathrm{mg}$ po & Olanzapine $5 \mathrm{mg}$ po \\
\hline & Tropisetron 5mg iv & Tropisetron $5 \mathrm{mg}$ iv & Tropisetron 5mg iv \\
\hline & Dexamethasone & Dexamethasone & Dexamethasone \\
\hline & $10 \mathrm{mg}$ po & $10 \mathrm{mg}$ po & $10 \mathrm{mg}$ po \\
\hline
\end{tabular}

\section{Follow-up}

Patients recorded and self-reported the times and dates of vomiting or retching episodes, and the use of rescue therapy from time of chemotherapy infusion $(0 \mathrm{~h})$ until day 5 . Patients were contacted in the mornings of days 2-5 to ensure compliance with nausea categorical scale. Functional Living IndexEmesis(FLIE) questionnaire scoring was self-administered early on the day5, directly following completion of final self-reports ${ }^{[11]}$. Notably, FLIE is a validated emesis- and nausea-specific questionnaire with nine nausea domain questions(items) and nine vomiting domain questions(items) and"no impact of CINV on daily life"represented means scores $>6$ on a 7-point scale $(>108 \text { in total })^{[12,13]}$.

All patients underwent post-treatment examination on days 6-8 and follow-up at days 19-21, and AEs related to aprepitant and olanzapine were recorded.

\section{Statistical analysis}

The sponsor managed the data and performed the analyses for this study. The hypothesis of this study was that the CR rate of $5 \mathrm{mg}$ olanzapine in quadruple group would be significantly higher than that of 5 mg olanzapine-based triplet group. Other trials have shown that the CR rate of triple antiemetic therapy was about $65 \%[14,15]$. According to the previous studies, CR rates of antiemesis treatment by olanzapine combined aprepitant, tropisetron and dexamethasone were $86 \%^{[14]}$. We believed that an improvement of more than $15 \%$ in the $\mathrm{CR}$ rate would be clinically meaningful. Therefore, assuming that the null hypothesis of the CR rate is $65 \%$ and the alternative hypothesis is $80 \%$, we calculated that a minimum of 82 patients were required to achieve a one-sided type I error of $0.1 \%$ and $80 \%$ of power, based on the exact binomial distribution. Because some dropouts were expected, we set the target sample size to 104, and the sample size calculation was performed by SASV.9.4 (Cary, NC, USA). 
Treatment comparisons were made using logistic regression models that included terms for treatment, gender, age, alcohol use, history of motion sickness etc. All comparisons used a two-sided significance level of $5 \%$. Tests of siginificance were based on the logistic regression models, and the norminal $P$ values were reported. Kaplan-Meier curves of time to first emesis were constructed to both groups. Fisher's exact test was used to compare the percentage of patients who got CR or experienced aprepitantrelated $A E s$ between the two groups.

\section{Results}

\section{Patients}

From March 2018 to March 2019, this prospctive, randomized, controlled study was conducted at the Medical Oncology Department of Ordos Central Hospital in Inner Mongolia, China. A total of 132 patients assigned to a study group with the use of random digits table. Six patients did not receive treatment (because of the cancellation of chemotherapy), and six patients dropped out of the study because of lacking of nausea data and FLIE questionnaires. Thus, 59 patients in the Quadruple group and 61 in the Triple group were completely assessed. The baseline characteristics were comparable between two groups(Table 2).

Table2 Patients'baseline characteristics [n(\%)] 


\begin{tabular}{|c|c|c|c|}
\hline Characteristics & $\begin{array}{l}\text { Quadruple Group } \\
(n=59)\end{array}$ & $\begin{array}{l}\text { Triple Group } \\
(n=61)\end{array}$ & $P$ \\
\hline \multicolumn{4}{|l|}{ Age(years) } \\
\hline$\geq 55$ & $60.39 \pm 9.22$ & $58.11 \pm 7.80$ & 0.104 \\
\hline Gender & & & 0.708 \\
\hline Female & $27(45.76)$ & $30(49.18)$ & \\
\hline Male & $32(54.24)$ & $31(50.82)$ & \\
\hline History of motion sickness & $9(15.25)$ & $14(22.95)$ & 0.284 \\
\hline $\begin{array}{l}\text { History of nausing with pregnancy in } \\
\text { female }\end{array}$ & $14(51.85)$ & $20(66.67)$ & 0.271 \\
\hline Alcohol use & & & 0.881 \\
\hline No Consumption & $32(54.24)$ & $32(52.46)$ & \\
\hline$<4$ drinks per week & $19(32.20)$ & $22(36.07)$ & \\
\hline$\geq 4$ drinks per week & $8(13.56)$ & $7(11.48)$ & \\
\hline Smoking Index & & & 0.144 \\
\hline No Smoking & $15(25.42)$ & $22(36.07)$ & \\
\hline $0 \sim 400$ & 10(16.95) & $4(6.56)$ & \\
\hline$\geq 400$ & $34(57.63)$ & $35(57.38)$ & \\
\hline Type of malignance & & & 0.850 \\
\hline Lung cancer & $31(52.54)$ & $31(50.82)$ & \\
\hline Others & $28(47.46)$ & $30(49.18)$ & \\
\hline Chemotherapy Cycle & & & 0.109 \\
\hline First- Cycle & $25(42.37)$ & $17(27.87)$ & \\
\hline Second to Third- Cycle & $22(37.29)$ & $22(36.07)$ & \\
\hline$\geq$ Fouth- Cycle & 12(20.34) & $22(36.07)$ & \\
\hline
\end{tabular}

\section{Efficacy}

The primary end-point of CR rate during the overall phase in the quadruple group(76.0\% (45/59)) was higher than those in the triple group $(67.0 \%(41 / 61))(P=0.271)$, but there was no statistic significance. During AP, the CR of quadruple group(100.0\%(59/59)) was significantly higher than triple group(93.0\% $(57 / 61))(P=0.045)$. The difference between the groups was especially greater in the delayed phase (24120 hours) (quadruple group $76.0 \%(45 / 59)$ vs triple group $67.0 \%(41 / 61)(P=0.271))$ (Figure 1). The total 
protection rates of quadruple group(28.8\% (17/59)) in the overall phase were also larger than triple group(23.0\%(14/61) $(P=0.463))$. During the $O P$, the incidence of no vomiting in quadruple group and triple group was $93.2 \%(55 / 59)$ vs $80.3 \%(49 / 61)(P=0.038)$, respectively. In the no-rescue treatment, few cases were reported in the quadruple group $(16.9 \%(10 / 59))$ than in the triple group group $(27.9 \%(17 / 61)$ $(P=0.152))$ during the OP.

\section{Comparison of FLIE Index}

According to FLIE, reports of no impact of CINV on daily life were exhibited by $47.5 \%(28 / 59)$ of the quadruple group and $44.3 \%(27 / 61)$ of the triple group $(P=0.035)$. The comparison of FLIE index of nause or vomiting between two groups was listed below in Table 3.

Table 3 Comparison of FLIE Index

\begin{tabular}{llcl} 
Items & Quadruple Group & Triple Group & $P$ \\
\hline Nausea FLIE Score & $48.92 \pm 12.32$ & $48.66 \pm 12.15$ & 0.907 \\
\hline Vomiting FLIE Score & $52.91 \pm 11.49$ & $50.67 \pm 13.05$ & 0.322 \\
\hline FLIE Score & $101.83 \pm 22.46$ & $99.33 \pm 24.70$ & 0.563
\end{tabular}

Notes $\mathbb{F L I E}$ : functional living index-emesis

\section{Comparison of time to first vomiting}

Kaplan-Meier curves of time to first emesis were obviously longer in the quadruple regimen group than that in the triple regimen $\operatorname{group}(P=0.031)$.

\section{Tolerability}

The most common aprepitant- and olanzapine-related AEs of the total patients were recorded. AEs included sedation, fatigue and constipation. The occurrences were observed in $57.6 \%(34 / 59), 54.2 \%$ (32/59)and $22.0 \%(13 / 59)$ of patients in the quadruple regimen group vs $50.8 \%(31 / 61), 52.5 \%(32 / 61)$ and $13.1 \%(8 / 61)$ in the triple regimen group $(P=0.454, P=0.854, P=0.199)$. The difference of AEs between two groups did not reach statistic significance. No grade 3 or 4 adverse events were obseaved in this study and no patients interrupted the study because of undesired sedation.

\section{Discussion}

Navari et al. conducted a Phase III study revealing that olanzapine $(10 \mathrm{mg})$ combined with standard therapy was superior at overall phase after chemotherapy, both at the primary endpoint (no nausea) and the secondary endpoint (CR rate) ${ }^{[14]}$. Olanzapine (10 mg) combined with NK-1RA, 5HT3-RA and dexamethasone quadruple therapy has been recommended antiemetic therapy for HEC in clinical guidelines of the Multinational Association of Supportive Care in Cancer/European Society for Medical 
Oncology(MASCC/ESMO), the American Society of Clinical Oncology(ASCO), and the National Comprehensive Cancer Network(NCCN $)^{[16]}$. However, the majority of trials involved antiemetic research have investigated patients receiving single-day cisplatin chemotherapy, and multiple-day chemotherapy (MDC) is one of the most neglected and challenging areas of antiemetic research due to the overlap of acute phase and delayed phase ${ }^{[1]}$.

We conducted this randomized, controlled, clinical trial enrolled patients who received 3-day cisplatinbased chemotherapy. It is more effective for combine $5 \mathrm{mg}$ olanzapine to triplet therapy (aprepitant, tropisetron, dexamethasone) versus $5 \mathrm{mg}$ olanzapine based triplet therapy in the early, later, and overall assessment phases. However, the primary and secondary end-points (CR rate) of OP and DP did not reach statistic significance, the secondary end-point $C R$ rate in $A P$ or no vomiting in $O P$ was significantly higher in the quadruple group. The primary end-point did not reach statistic significance in this study which was inconsistent with previous reports of Navari et al ${ }^{[14]}$. The possible explanations for those differences may be complex. On the one hand, we designed $5 \mathrm{mg}$ olanzapine rather than NK-1 receptor antagonist based triplet therapy as the control group because a phase 3 trial showed that olanzapine 10 $\mathrm{mg}$ was comparable to aprepitant in the control of CINV ,but somnolence is a major side effection ${ }^{[6]}$. And olanzapine $5 \mathrm{mg}$ has shown equivalent activity to olanzapine $10 \mathrm{mg}$ and a favourable safety in relation to somnolence in phase 2 study, thus it is reasonable for this clinical design ${ }^{[7]}$. On the other hand, we adopted $\mathrm{CR}$ as primary end-point that was different from previous quadruple regimen clinical studies which adopted no nausea or TC as primary end point ${ }^{[14,15]}$. As we know, nausea was better controlled by olanzapine in delayed period and overall period, so it may explained that the TC rates were identical in both groups in our study ${ }^{[6]}$. Besides these, a randomized, double-blind, placebo-controlled, phase 3 study enrolled 710 patients to evaluate the efficacy of olanzapine $5 \mathrm{mg}$ with triplet antiemetic therapy adopted $\mathrm{CR}$ in the delayed phase as primary endpoint. The proportion of patients who achieved a complete response was $79 \%$ vs $66 \%(P<0.0001)^{[8]}$, and our clinical results of $76 \%$ vs $67 \%$ were highly accordence with this phase 3 study, and a relative smaller sample size in this study than those in phase III trial maybe one of the reasons of not reaching statistic significantly in OP and $D P^{[8,14]}$. Furthermore, the different doses of 5-HT3RA, DXM, olanzapine and given number of days in the study also affected the results of those quadruple vs triplet regimen comparison studies ${ }^{[8,14]}$. This study and J-FORCE both adopted $5 \mathrm{mg}$ olanzapine achieved higher CR rate compared with a phase III study which adopted $10 \mathrm{mg}$ olanzapine $\mathrm{e}^{[8,}$ 14]. The different time cut-off points between acute phase and delayed phase in multiple-day cisplatininduced chemotherapy may affect the CR rate, as shown by H.F.Gao that CR declined about $20 \%$ when acute phase cut-off point switched from 24 hour to 72 hour ${ }^{[17]}$. We defined 24 hour as acute phase in this study and $25 \%$ enrolled patients received $<70 \mathrm{mg} / \mathrm{m}^{2}$ cisplatin in J-FORCE study may explained the higher $\mathrm{CR}$ in some degree. This also supports the previously conclusion that olanzapine $5 \mathrm{mg}$ has equivalent activity to $10 \mathrm{mg}$ in CINV prevention ${ }^{[7,8]}$. The superiority of $5 \mathrm{mg}$ olanzapine-based quadruple regimen in the study was also support its clinical advantages for prevention of CINV induced by cisplatin multipleday chemotherapy. 
According to FLIE, no impact of CINV on daily life was defined as questionnaire total score $>108$, this study exhibited identical life quality in quadruple regimen group to the triplet regimen group. Kaplan-Meier curves of time to first emesis in the quadruple regimen group were obviously longer than the triple regimen group $(P=0.031)$ and this was in accordance with no vomiting in OP which was significantly higher in the quadruple group. This supports the superiority of control of chemotherapy-induced vomiting $(\mathrm{CIV})$ by $5 \mathrm{mg}$ olanzapine-based quadruple regimen induced by cisplatin multiple-day chemotherapy.

The most common aprepitant- and olanzapine-related AEs included sedation, fatigue and constipation. The occurrences between two groups were identical and it were consistent with other studies

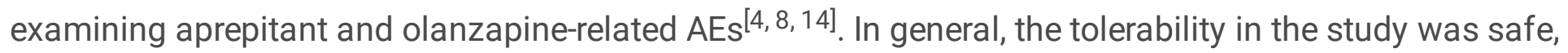
no grade 3 or 4 adverse events were observed in this study, and no patients discontinued the study because of undesired sedation.

\section{Conclusion}

In summary, $5 \mathrm{mg}$ olanzapine plus triplet therapy (aprepitant, tropisetron, dexamethasone) is more effective than 5-mg olanzapine-based triplet therapy for the prevention of CINV induced by multiple-day cisplatin chemotherapy. Clinical studies with larger sample sizes were needed to further confirm it and $5 \mathrm{mg}$ olanzapine combined with new generation of 5-HT3RA palonosetron and NK-1RA fosaprepitant for the prevention of CINV induced by multiple-day cisplatin chemotherapy clinical study is ongoing.

\section{Declarations}

Funding: This study was supported by Ordos Health Commission Key discipline Project, Inner Mongolia Medical University Science and Technology million Project funding,Ordos science and technology project

Conflicts of interest/Competing interests: The authors declare that they have no known competing financial interests or personal relationships that could have appeared to influence the work reported in this paper.

Availability of data and material: All data generated or analysed during this study are included in this published article [and its supplementary information files]

Code availability: Not applicable.

Authors' contributions: Gaowa Jin participated in supervision,project administration and funding acquisition.Quanfu Li participated in writing - original draft,conceptualization,methodology,funding acquisition,software.Jiali Gao participated in investigation,formal analysis and data curation. Researchers of the study investigation included Jun Zhao,Caihong Jiang,Feng Chen,Lanzhen Zhao,Ying Jiang,Hui Li,Wenjuan Wang,Yungaowa Wu,Yilan Jin,Lenggaowa Da,Yajuan Zhang and Hongxia Li.Zewei Zhang was responsible for Software 
Ethics approval: All procedures performed in studies involving human participants were in accordance with the ethical standards of the institutional and/or national research committee and with the 1964 Helsinki Declaration and its later amendments or comparable ethical standards. The study was approved by the Bioethics Committee of the Ordos Central Hospital.

Consent to participate: Informed consent was obtained from all individual participants included in the study.

Consent for publication: Written informed consent was obtained from the patient for publication of this article and any accompanying images. A copy of the written consent is available for review by the Editorin-Chief of this journal.

\section{References}

1. Herrstedt Jørn., Roila Fausto., Warr David., Celio Luigi., Navari Rudolph M., Hesketh Paul J., Chan Alexandre., Aapro Matti S.(2017). 2016 Updated MASCC/ESMO Consensus Recommendations: Prevention of Nausea and Vomiting Following High Emetic Risk Chemotherapy. Support Care Cancer, 25(1), 277-288. doi:10.1007/s00520-016-3313-0,

2. Hesketh PJ, Bohlke K, Lyman GH. Antiementics $₫$ Americian Society of Clinical Oncology Focused Guideline Update. J Clin Oncol 2016, 34, 381-386.

3. Albany C, Brames MJ, Fausel C et al. Randomized, double-blind, placebo controlled phase III crossover study evaluating the oral neurokinin-1 antagonist aprepitant in combination with a 5HT3 receptor antagonist and dexamethasone in patients with germ cell tumors receiving 5 day-cisplatin combination chemotherapy regimens: a Hoosier Oncology Group (HOG) study. J Clin Oncol2012, 30: 3998-4003.

4. Li Q, Wang W, Chen G, et al.Evaluation of a Neurokinin-1 Antagonist in Preventing Multiple-day Cisplatin-induced Nausea and Vomiting.Open Med (Wars). 2018 Mar 15,13:29-34.

5. Ng TL, Hutton B, Clemons M. Chemotherapy-induced nausea and vomiting:time for more emphasis on nausea? Oncologist 2015, 20: 576-83.

6. Navari RM, Gray SE, Kerr AC. Olanzapine versus aprepitant for the prevention of chemotherapyinduced nausea and vomiting: a randomized phase III trial. J Support Oncol 2011, 9: 188-95.

7. Yanai Takako., Iwasa Satoru., Hashimoto Hironobu., Ohyanagi Fumiyoshi., Takiguchi Tomomi., Takeda Koji., Nakao Masahiko., Sakai Hiroshi., Nakayama Toshiaki., Minato Koichi., Arai Takahiro., Suzuki Kenichi., Shimada Yasuhiro., Nagashima Kengo., Terakado Hiroyuki., Yamamoto Noboru. (2018). A double-blind randomized phase II dose-finding study of olanzapine $10 \mathrm{mg}$ or $5 \mathrm{mg}$ for the prophylaxis of emesis induced by highly emetogenic cisplatin-based chemotherapy. Int J Clin Oncol, 23(2), 382-388. doi:10.1007/s10147-017-1200-4

8. Hashimoto Hironobu., Abe Masakazu., Tokuyama Osamu., Mizutani Hideaki., Uchitomi Yosuke., Yamaguchi Takuhiro., Hoshina Yukari., Sakata Yasuhiko., Takahashi Takako Yanai., Nakashima Kazuhisa., Nakao Masahiko., Takei Daisuke., Zenda Sadamoto., Mizukami Koki., Iwasa Satoru., 
Sakurai Michiru., Yamamoto Noboru., Ohe Yuichiro.(2020). Olanzapine $5 \mathrm{mg}$ plus standard antiemetic therapy for the prevention of chemotherapy-induced nausea and vomiting (J-FORCE): a multicentre, randomised, double-blind, placebo-controlled, phase 3 trial. Lancet Oncol, 21(2), 242-249. doi:10.1016/S1470-2045(19)30678-3

9. Patel Priya., Leeder J Steven., Piquette-Miller Micheline., Dupuis L Lee.(2017). Aprepitant and fosaprepitant drug interactions: a systematic review. Br J Clin Pharmacol, 83(10), 2148-2162. doi:10.1111/bcp.13322

10. McCrea JB, Majumdar AK, Goldberg MR, et al. Effects of the neurokinin-1 receptor antagonist aprepitant on the pharmacokinetics of dexamethasone and methylprednisolone[J]. Clin Pharmacol Ther, 2003, 74(1): 17-24.https://doi.org/10.1016/S0009-9236(03)00066-3.

11. Martin AR, Pearson JD, Cai B, et al. Assessing the impact of chemotherapy-induced nausea and vomiting on patients' daily lives: a modified version of the Functional Living Index-Emesis (FLIE) with 5-day recall[J]. Support Care Cancer, 2003, 11(8): 522-7.

12. Decker GM, DeMeyer ES, Kisko DL. Measuring the maintenance of daily life activities using the functional living index-emesis (FLIE) in patients receiving moderately emetogenic chemotherapy[J]. J Support Oncol, 2006, 4(1): 35-41, 52.

13. Martin AR, Carides AD, Pearson JD, et al. Functional relevance of antiemetic control:experience using the FLIE questionnaire in a randomised study of the NK-1 antagonist aprepitant[J]. Eur $\mathrm{J}$ Cancer, 2003, 39(10): 1395-401.

14. Navari R M, Qin R,Ruddy KJ, et al. Olanzapine for the Prevention of Chemotherapy-Induced Nausea and Vomiting[J]. New England Journal of Medicine, 2016, 375(2):134-142.

15. Mizukami N, Yamauchi M , Koike K, et al. Olanzapine for the Prevention of Chemotherapy-Induced Nausea and Vomiting in Patients Receiving Highly or Moderately Emetogenic Chemotherapy: A Randomized, Double-Blind, Placebo-Controlled Study[J]. Journal of Pain \& Symptom Management, 2014, 47(3):542-550.

16. Razvi Y, Chan S, Mcfarlane T, et al. ASCO, NCCN, MASCC/ESMO: a comparison of antiemetic guidelines for the treatment of chemotherapy-induced nausea and vomiting in adult patients[J]. Supportive Care in Cancer,2019,27(1):87-95.

17. Gao HF, Liang Y, Zhou NN et al. Aprepitant plus palonosetron and dexamethasone for prevention of chemotherapy-induced nausea and vomiting in patients receiving multiple-day cisplatin chemotherapy[J]. Intern Med J.2013 Jan,43(1):73-76.

\section{Figures}




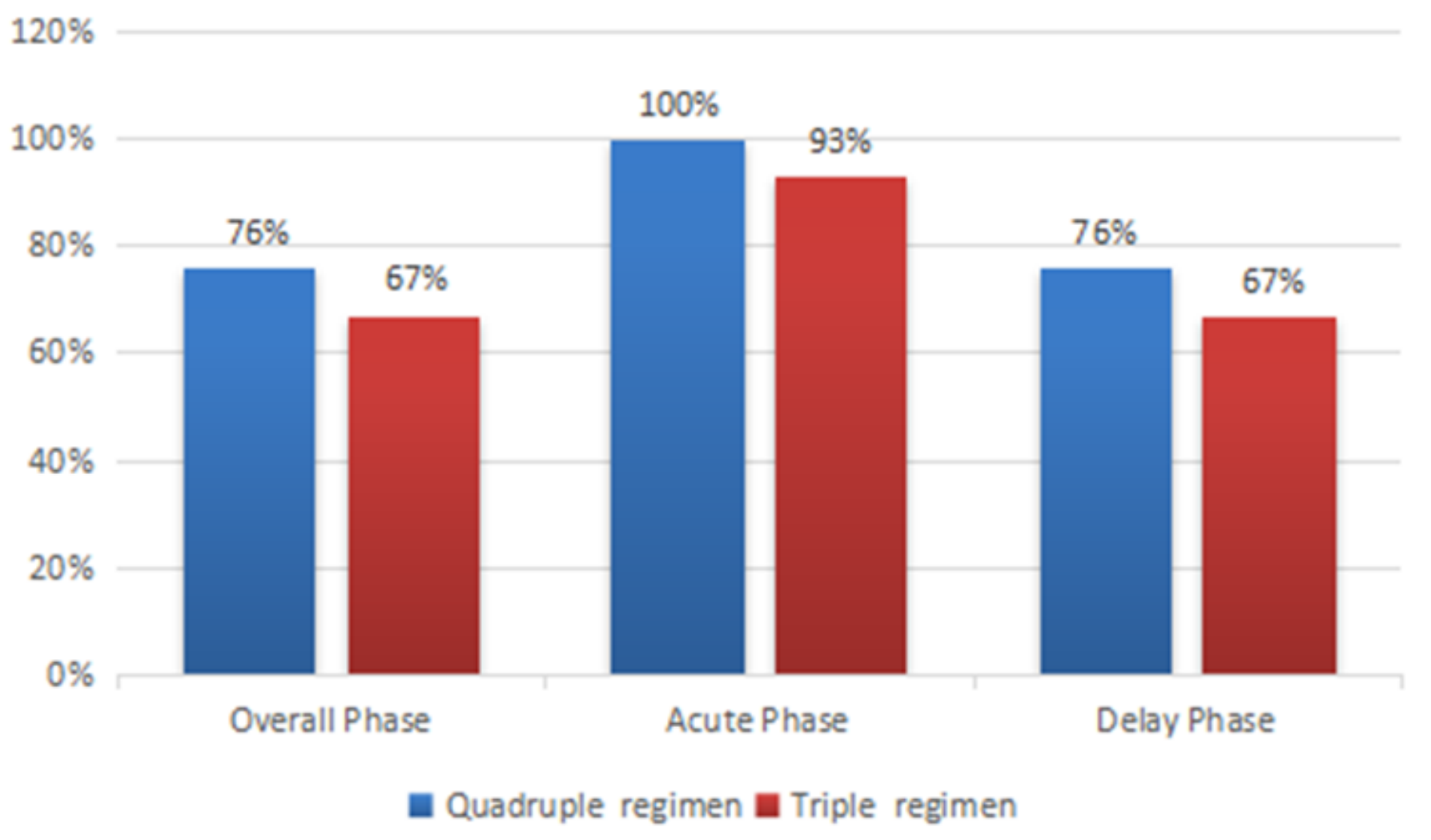

Figure 1

Comparison of Complete Response Between Two Groups 


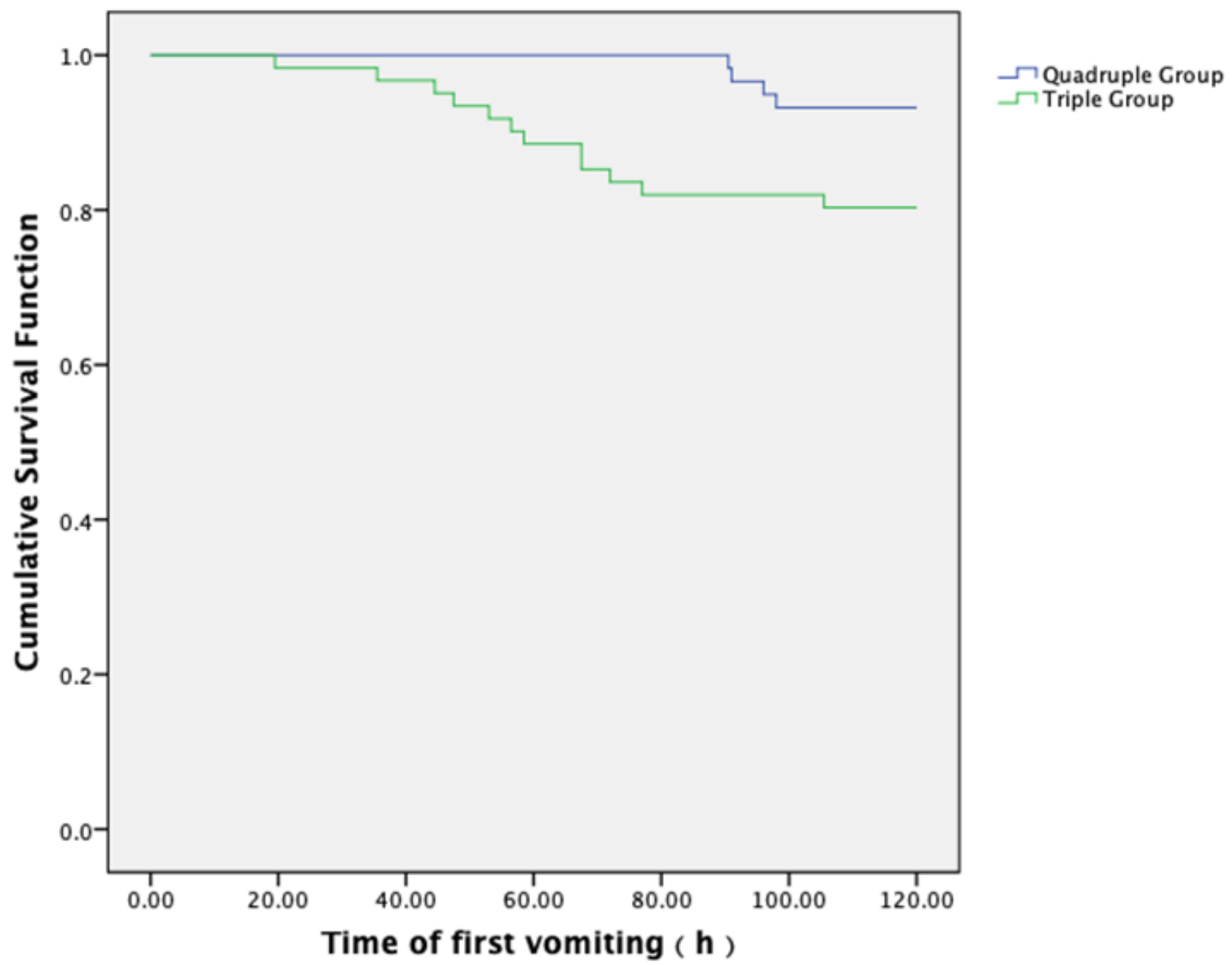

Figure 2

Comparison of time to first vomiting between two groups 